\title{
Influence of Electric Field on the Orientation of Ferroelectric Liquid Crystals inside micro capillaries
}

\author{
Daniel Budaszewski, * Andrzej W. Domański, and Tomasz R. Woliński \\ Faculty of Physics, Warsaw University of Technology, Koszykowa 75, 00-662 Warszawa,
}

Received February 24, 2013; accepted March 25, 2013; published March 31, 2013

\begin{abstract}
In the paper we present recent results on investigation of ferroelectric liquid crystals molecules orientation inside photonic crystal fibers, which consist of a set of micro capillaries. We have infiltrated glass micro capillaries of different inner diameters with the experimental ferroelectric liquid crystal mixture W-206K. The orientation of the liquid crystal molecules was controlled by an external electric field applied to the sample. We have obtained promising results about the orientation of the ferroelectric liquid crystal molecules and their behavior under the influence of the applied external electric field. The presented results may lead to designing a new class of fast-switching fiber optic sensors.
\end{abstract}

One of the attractive properties of photonic crystal fibers (PCF) [1] is that their air-hole lattice structure can be infiltrated with substances whose refractive index can be controlled by external physical fields. The infiltration of PCFs with different types of liquid crystal (LC) materials forms a new class of tunable optical fibers that has been investigated by many research groups all over the world. Experimental evidence of tuning properties of photonic liquid crystal fibers has been reported since 2003 [2-4], but so far the vast majority of work has been done by employing nematic liquid crystals (NLC) and only the initial study included cholesterics and smectics.

Chiral smectic C (Sm C*) liquid crystals possess very fast electro-optical response on external electric fields, c. an order of magnitude faster than commonly used nematic liquid crystals. Other properties of $\mathrm{Sm} \mathrm{C}^{*}$ worth mentioning are bistability, optical memory and large viewing angles when they are applied in displays. Thanks to their properties ferroelectric liquid crystals (FLC) remain promising candidates for many technological applications, especially in modern types of liquid crystal displays as well as in fiber optic sensors and tuneable telecommunication filters. The molecules of SmC* liquid crystals are arranged in a helical twist mainly due to the presence of a chiral dopant. The electro-optical behaviour of FLCs depends mainly on few parameters as a tilt angle, spontaneous polarization, helical pitch, elastic properties, dielectric and optical anisotropy and anchoring energy. All these parameters are responsible for the alignment of FLC molecules inside the surrounding glass cells. Unlike

*E-mail: danielb@if.pw.edu.pl nematics it is very difficult to obtain a uniform FLC orientation without any defects. In general, FLC layers tend to orient perpendicularly to the glass cells forming book-shelf geometry. However, it is observable in many cases that the alignment is full of zig-zag defects that result from the FLC nature. There exists a novel aligning technique that allows avoiding this kind of defects [5].

The most interesting electro-optical effects in FLCs are a deformed helix ferroelectric (DHF) mode [6] and an electrically suppressed mode (ESH) $[7,8]$, both based on photo-alignment methods. The mentioned modes may find their application also in PCF's. The main advantages of DHF mode are the insensitivity to voltage polarity, and even two orders faster response time in comparison to NLC, which is, in addition, frequency dependent. This mode is observable for driving voltages below the critical voltage necessary for helix unwinding. In the ESH mode the FLC helix is suppressed by the influence of an external electric field with a voltage above the critical value. This mode is, in general, similar to a surface stabilized FLC (SSFLC) invented by Clark and Lagerwall [9], but in this case the helix is suppressed by glass cell surfaces. The advantage of the ESH mode is its very fast response time.

Recently, a new research line has been started by infiltrating the microstructured PCFs with FLC. Since ferroelectric liquid crystals possess a very fast electrooptical response when influenced by electric fields [10, 11] they are a promising candidate for novel tuneable optical filters and sensors. Unlike FLC layers between two glass plates, in micro capillaries we have cylindrical geometry and hence the alignment of the FLC molecules may be different from those observed in classical LC cells. We have investigated the orientation of the FLC molecules inside the micro capillaries with different inner diameters varying from $1 \mu \mathrm{m}$ up to $30 \mu \mathrm{m}$. The glass micro capillaries were manufactured at the Maria CurieSklodowska University (UMCS), Lublin, Poland.

In the following studies we have used micro capillaries with and without a thermo aligning planar layer, which were obtained by using a commercially available polyimide substance SE-130 manufactured by Nissan Chemicals. 
To prepare a thermo orienting layer inside the micro capillary at first we infiltrated the structure with SE-130 polyimide, along the whole length, using high pressure air. Next, the micro capillary was baked in a temperature of $130^{\circ} \mathrm{C}$ to remove redundant polyimide from the micro capillary. As a result, we obtained a planar aligning layer inside the structure.

The micro-capillaries were infiltrated with an $\mathrm{SmC}^{*}$ mixture W-206K synthetized at the Military University of Technology (MUT). According to the datasheet of this mixture, the helical pitch was higher than $2000 \mu \mathrm{m}$, the tilt angle was 30 degrees and the spontaneous polarization was $31 \mathrm{nC} / \mathrm{cm}^{2}$, all of them measured in a temperature of $25^{\circ} \mathrm{C}$. The phase transitions sequence of the FLC is: $\mathrm{SmC}^{*}\left(75^{\circ} \mathrm{C}\right) \mathrm{SmA}\left(90^{\circ} \mathrm{C}\right) \mathrm{N}\left(93.5-97.9^{\circ} \mathrm{C}\right)$ Iso.

To infiltrate the micro-capillaries with FLC we used our experimental device for infiltrating the PCFs with LC in high temperatures (Fig.1). Both the micro-capillary and the FLC were heated up to the isotropic phase which was necessary to infiltrate the structure by using a capillary forces method.

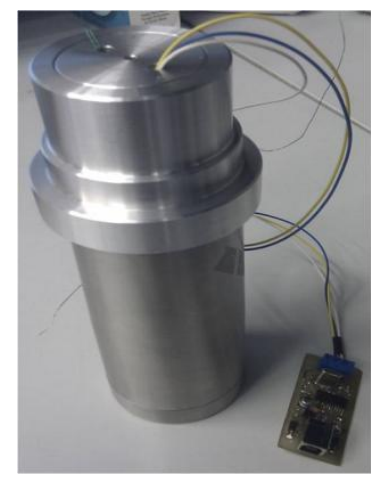

Fig.1. Chamber for PCF infiltrating with LC in high temperatures.

Each of the infiltrated micro capillaries was put into an oven and heated again up to the isotropic phase, and next slowly cooled down with a speed of $1^{\circ} \mathrm{C} / \mathrm{min}$. in order to align FLC molecules.

We observed the resulting orientation of FLC molecules inside the micro capillary placed on a rotating table under a polarizing microscope with crossed polarizers.

For the micro capillary with an inner diameter of $3 \mu \mathrm{m}$ we observed a bright state for one position of the table and a dark state for a perpendicular position. This means that we obtained a planar orientation of molecules inside the micro capillary (Fig. 2).

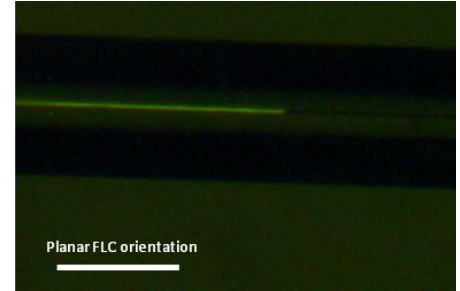

Fig. 2. A 3- $\mu \mathrm{m}$ micro capillary partially infiltrated with FLC W $206 \mathrm{~K}$ (left side).

An interesting effect was observed for a $6 \mu \mathrm{m}$-diameter micro capillary without orienting layer filled with $\mathrm{W}$ 206K FLC. We obtained a planar orientation on one part of the micro capillary (Fig. 3a) and a helical structure on the next part (Fig. 3b)
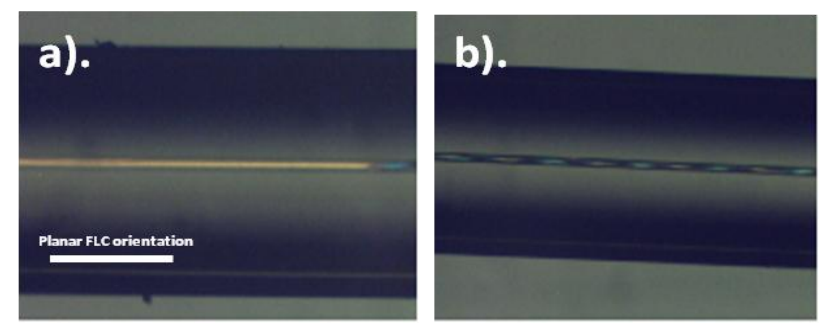

Fig. 3. A $6 \mu \mathrm{m}$ micro capillary without orienting layer infiltrated with W $206 \mathrm{~K}$ FLC. a) planar orientation of the molecules b) helical orientation of the FLC molecules.

To confirm the hypothesis of helical orientation of the molecules we had been rotating the investigated micro capillary along its axis under a polarizing microscope. We noticed that by rotating the sample the dark regions were moving in one direction. This effect was spontaneous and one of the potential explanations is that during the cooling process of the FLC we obtained a planar orientation induced by flow.

In our research we have also investigated influence of external electric field on switching of FLC molecules inside micro capillaries. The experimental setup is shown in Fig. 4.

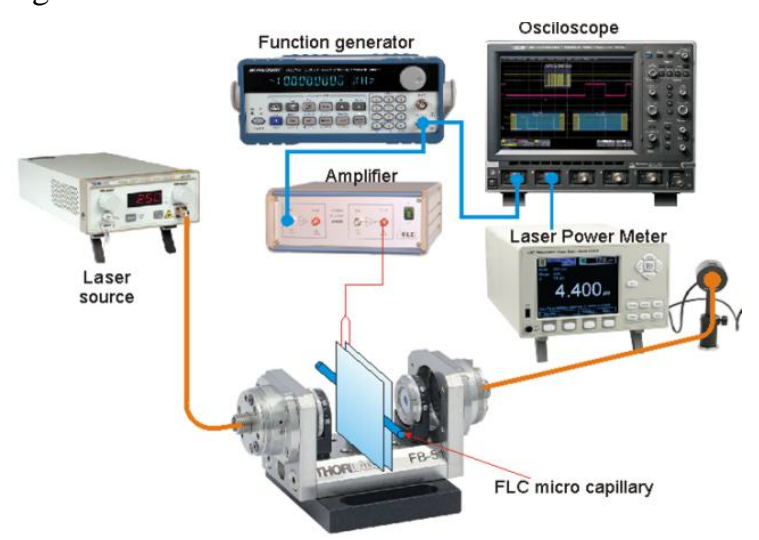

Fig. 4. Experimental setup for investigating the switching of the FLC molecules in micro capillaries under influence of external electric field. 
The investigated part of the micro capillary was placed between two glass plates covered with a conducting ITO layer. Next, we placed the sample between two crossed polarizers. As a light source we used a laser diode working on the wavelength $\lambda=1550 \mathrm{~nm}$. The signal was registered by a high-sensitive optical power meter NEWPORT 2936 connected to a digital oscilloscope LeCROY. The external electric field was generated by a function generator connected to an amplifier. We applied a square-shape signal from the function generator with an amplitude of $8 \mathrm{~V}$, which was next amplified 200 times in order to switch the FLC molecules inside the micro capillary. As a result, the voltage between the two electrodes was $1600 \mathrm{~V}$. The frequency of the signal was varying from $1 \mathrm{~Hz}$ up to $100 \mathrm{~Hz}$. We observed fast relaxation of the FLC molecules for low frequencies of the external electric field (Fig. 5).

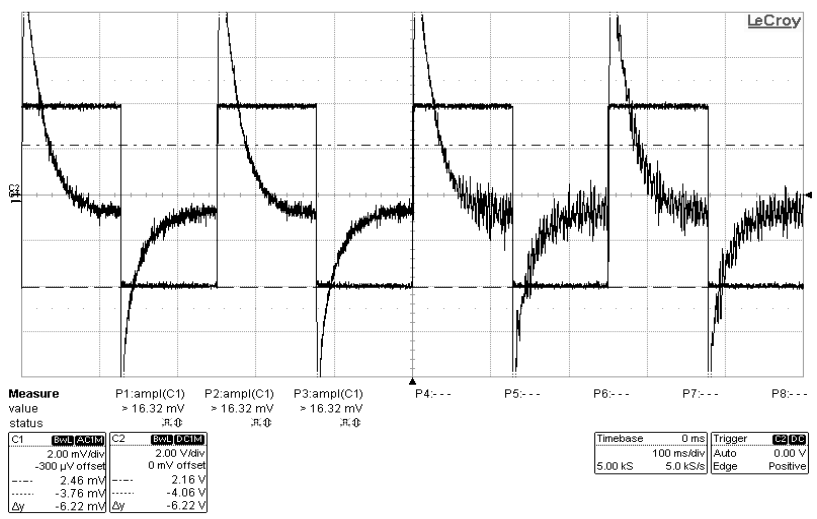

Fig. 5. Response time and time of relaxation of the FLC molecules for square-shaped external electric field with frequency of $7 \mathrm{~Hz}$.

We measured the response time of the sample by analysing the view from the digital oscilloscope. The response time $\tau_{0.1-0.9}$ defined as the switching time between $10 \%$ and $90 \%$ of light transmittance was c.a. $1 \mathrm{~ms}$. The switching time of the FLC molecules inside the micro-capillaries could be improved by decreasing the distance between electrodes. Also further studies on other FLC substances with larger dielectric anisotropy or lower rotational viscosity may be necessary to analyze the response time as well as the contrast ratio in the microcapillaries.

To conclude, we have investigated the orientation of FLC molecules inside of micro capillaries of different diameters, and we have obtained in one case even a helical orientation of the molecules. However, there is a need for further research to explain this phenomenon. Additionally, we have analysed the influence of an external electric field on the orientation of the FLC molecules and their switching times. We have noticed that for low frequency electric fields the FLC molecules tend to their original positions and moves only when the sign of the signal is changing. Hence, to keep the FLC molecules in one position we need to control them with higher frequencies of the applied electric field.

Although our results consider only the behaviour of FLC molecules inside single capillaries, they can be also extended to PCFs. which may result in designing new fast-switching optical filters, attenuators and electric field sensors. One of the benefits of using FLCs in comparison with NLC is their very fast response time, and also new molecular orientations like in DHF or ESH modes.

The authors would like to acknowledge Prof. Roman Dąbrowski from the Military University of Technology for FLC mixtures and Dr. Paweł Mergo from the Maria Curie Skłodowska University for the micro-capillaries samples.

This work is supported by the National Science Centre under the grant No. 2011/03/B/ST7/02547

\section{References}

[1] P.St.J. Russell, J. Light. Technol. 24, 4729 (2006).

[2] T. Larsen, A. Bjarklev, D. Hermann, J. Broeng, Opt. Exp. 11, 2589 (2003).

[3] T.R. Wolinski, K. Szaniawska, K. Bondarczuk, P. Lesiak, A.W. Domanski, R. Dabrowski, E. Nowinowski-Kruszelnicki, J. Wojcik, Opto-Electronics Rev. 13(2), 59 (2005).

[4] M.W. Haakestad et al., IEEE Photon. Technol. Lett. 17, 819 (2005).

[5] M. Matuszczyk, Structures and Properties of Ferroelectric Liquid Crystal Displays (Chalmers Reproservice, Goteborg, Sweden 1996).

[6] Q. Guo, E.P. Pozhidaev, A.K. Srivastava, Y. Ma, X. Wang, V.G. Chigrinov, H.S. Kwok, SID Symposium Digest of Technical Papers, 43(1), 449 (2012).

[7] E.P. Pozhidaev et al., Proc. of 31-th International Display Research Conference EuroDisplay 2011, Bordeaux-Arcachon, France, September 19-22 (2011).

[8] V. Chigrinov, E. Pozhidaev, A. Srivastava, G. Qi, M. Ying, H.S. Kwok, Fast Ferroelectric Liquid Crystal Modes Based on Photoaligning Technology (SID 2013,Vancouver, Canada, May 2013).

[9] N.A. Clark, S.T. Lagerwall, Appl. Phys. Lett. 36, 899 (1981).

[10] S. Mathews, Y. Semenova, G. Rajan, G. Farrell, Proc. SPIE 7316, 73161E (2009).

[11] D. Budaszewski, T.R. Woliński, M.A. Geday, J.M. Otón, Phot. Lett. Poland 2, 3 (2010). 